\title{
The Role of Optical Coherence Tomography in an Atypical Case of Oculocutaneous Albinism: A Case Report
}

\author{
Settimio Rossi ${ }^{a} \quad$ Francesco Testa $^{a}$ Annagiusi Gargiulo ${ }^{b}$ \\ Valentina Di lorio ${ }^{a}$ Raffaella Brunetti Pierri ${ }^{a}$ \\ Francesco Maria D'Alterio ${ }^{a}$ Michele Della Corte $^{a}$ \\ Enrico Surace $^{\mathrm{b}}$ Francesca Simonelli $^{\mathrm{a}}$ \\ ${ }^{a}$ Department of Ophthalmology, Second University of Naples, and \\ ${ }^{\mathrm{b}}$ Telethon Institute of Genetics and Medicine (TIGEM), Naples, Italy
}

\section{Key Words}

Oculocutaneous albinism · Optical coherence tomography · Atypical albinism

\begin{abstract}
Background: Oculocutaneous albinism is a group of autosomal recessive disorders featuring hypopigmentation of the hair, skin and eyes. Ocular signs associated with the disease are nystagmus, decreased visual acuity, hypopigmentation of the retina, foveal hypoplasia, translucency of the iris, macular transparency, photophobia and abnormal decussation of nerve fibers at the chiasm.

Case Report: An 8-year-old Caucasian girl presented to our clinic 'Referral Center for Hereditary Retinopathies' of the Second University of Naples with a diagnosis of Stargardt disease and a progressive reduction in visual acuity in both eyes. She underwent a complete ophthalmic examination including standard electroretinography and optical coherence tomography (OCT). A molecular analysis was also performed. Best-corrected visual acuity was 20/30 in the right eye and 20/40 in the left eye. Biomicroscopy of the anterior segment revealed a transparent cornea, in situ and transparent lens and normally pigmented iris. A mild diffuse depigmentation and macular dystrophy were observed at fundus examination. Standard electroretinography showed normal scotopic and photopic responses. OCT revealed high reflectivity across the fovea without depression. The typical OCT pattern led us to direct the molecular analysis towards the genes involved in oculocutaneous albinism. The molecular analysis identified mutations in the TYR gene.
\end{abstract}

Conclusion: In this case, the role of OCT was crucial in guiding the molecular analysis for the diagnosis of albinism. OCT is therefore instrumental in similar cases that do not present 
typical characteristics of a disease. The case also proves the relevance of molecular analysis to confirm clinical diagnoses in hereditary retinal diseases.

\section{Background}

Oculocutaneous albinism (OCA) is a group of autosomal recessive disorders featuring hypopigmentation of the hair, skin and eyes. Approximately 1 in 17,000 people suffers from OCA. To date, four types of OCA (OCA1-4), attributed to mutations in TYR, OCA2, TYRP1 and MATP, respectively, have been identified. Ocular signs associated with the disease are nystagmus, decreased visual acuity, hypopigmentation of the retina, foveal hypoplasia, translucency of the iris, macular transparency, photophobia and abnormal decussation of nerve fibers at the chiasm [1].

This article describes an atypical case of OCA in which the use of optical coherence tomography (OCT) was crucial for establishing the diagnosis.

\section{Case Report}

An 8-year-old Caucasian girl presented to our clinic 'Referral Center for Hereditary Retinopathies' of the Second University of Naples. The patient complained of a progressive reduction in visual acuity in both eyes, leading to the diagnosis of Stargardt disease. She had no past ocular history of trauma, surgery or inflammatory disease. Her past medical history was significant for exotropia and visual acuity impairment.

On examination, visual acuity was $20 / 30$ in the right eye and 20/40 in the left eye. The orthoptic examination showed the presence of exoforia. Biomicroscopy of the anterior segment revealed a transparent cornea, in situ and clear lens and normally pigmented iris in both eyes. A slightly diffuse depigmentation in the midperiphery and a pinkish optic disk with clear margins and mild macular dystrophy at the posterior pole were observed at fundus examination.

Standard electroretinography showed normal scotopic and photopic responses. OCT revealed the absence of normal foveal depression (foveal thickness was $295 \mu \mathrm{m}$ in the right eye and $299 \mu \mathrm{m}$ in the left eye; normal range 168-239 $\mu \mathrm{m}$ ) and of the small elevation of the inner segment/outer segment junction in the fovea. Furthermore, the high reflectivity across the fovea suggested a persisting nerve fiber layer and multiple inner retinal layers across the fovea (fig. 1). Hence, all OCT findings were in contrast with the diagnosis of juvenile macular dystrophy [2,3]. The clinical pattern of foveal hypoplasia revealed by OCT was instead similar to that observed in albinism [4, 5], even if the patient featured normal pigmentation of the skin, hair and iris (fig. 2) and did not show typical ocular albinism signs such as nystagmus and iris transillumination. A molecular analysis was then performed on the genes involved in albinism and revealed the presence of R402Q and 1177delG mutations in the TYR gene.

This research was carried out in compliance with the Helsinki Declaration. Written consent was obtained from the patient for the publication of this case report and the accompanying images. A copy of the written consent is available for review by the Editor-in-Chief of this journal.

\section{Conclusions}

OCT has been shown to provide detailed morphometric information on retinal structure and to be an important tool for the diagnosis and follow-up of diseases such as age-related macular degeneration, choroidal neovascularization and macular edema 
[6-8]. In recent years, the use of this technique has been applied to other types of retinal diseases aiding in the differential diagnosis of hereditary retinal dystrophies. Today, OCT has become an important examination method for the diagnosis and followup of retinitis pigmentosa and its complications [9] and can be considered a marker in cases of Stargardt disease [2,3] and albinism [4, 5].

In this case report, OCT was crucial in guiding the molecular analysis for the diagnosis of albinism. OCT can therefore be considered an important diagnostic tool in cases that do not present typical ocular and cutaneous characteristics of a disease.

In fact, OCT showed a thicker fovea in our patient compared to the general population, which may be due to the absence of a foveal pit as part of foveal hypoplasia associated with OCA [10]. Moreover, the OCT images revealed the absence of the small elevation of the inner segment/outer segment junction in the fovea and the persistence of a nerve fiber layer and multiple inner retinal layers across the fovea; these two features may be explained by the foveal hypoplasia as previously described in the studies by Craft et al. [11] and Chong et al. [4] relating these features to foveal hypoplasia in OCA patients.

Our case also proves the relevance of molecular analysis to confirm clinical diagnoses in hereditary retinal diseases. Furthermore, the phenotype described in this report suggests that patients affected by OCA associated with R402Q and 1177delG TYR gene mutations may present less severe clinical manifestations including good visual acuity, absence of nystagmus and an evident iris, skin and hair pigmentation.

In conclusion, this case confirms the relevance of OCT in the diagnosis of hereditary retinal dystrophies, providing characteristic patterns that can be considered reliable disease markers.

\section{Disclosure Statement}

The authors declare that they have no competing interests. 

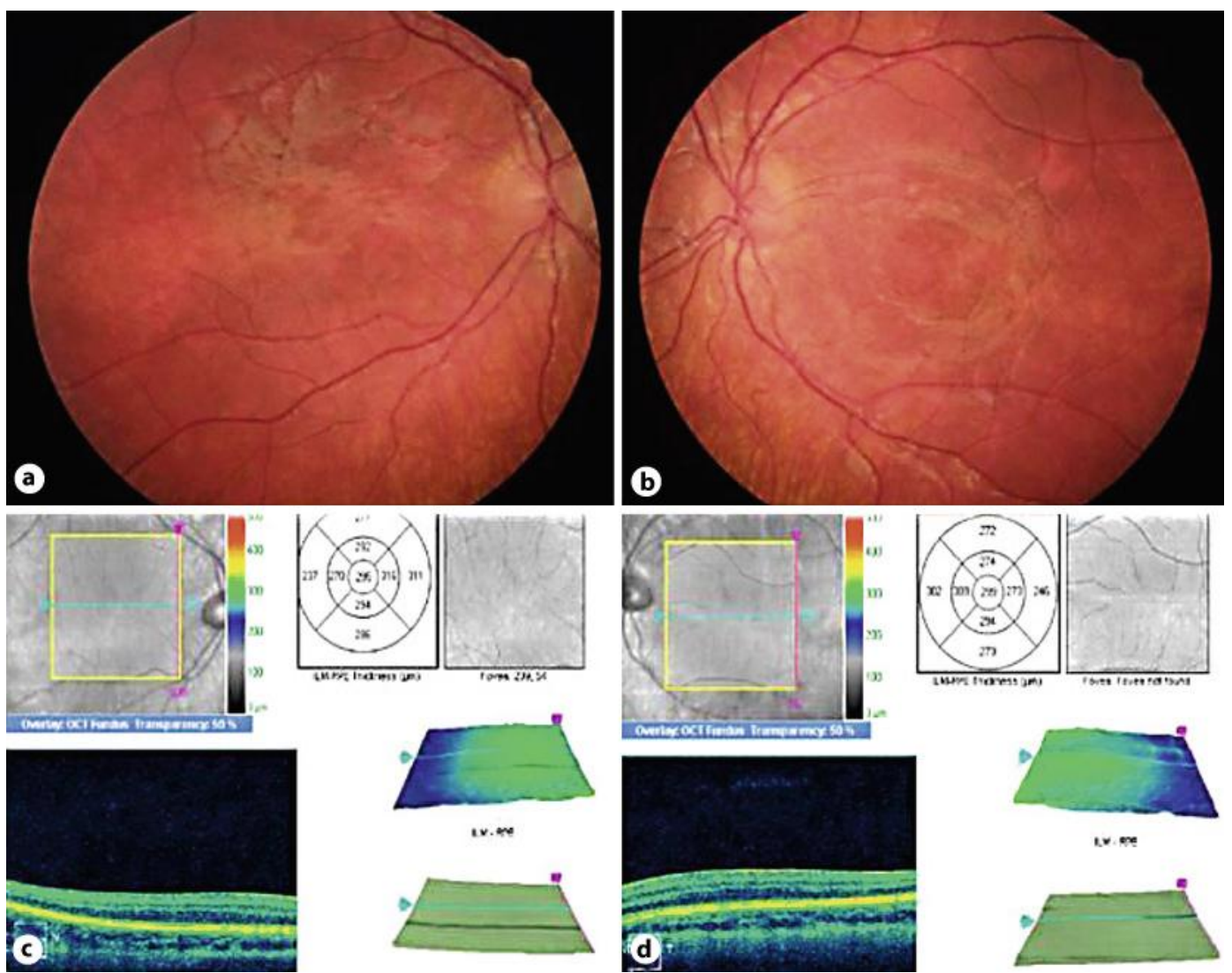

ix.me
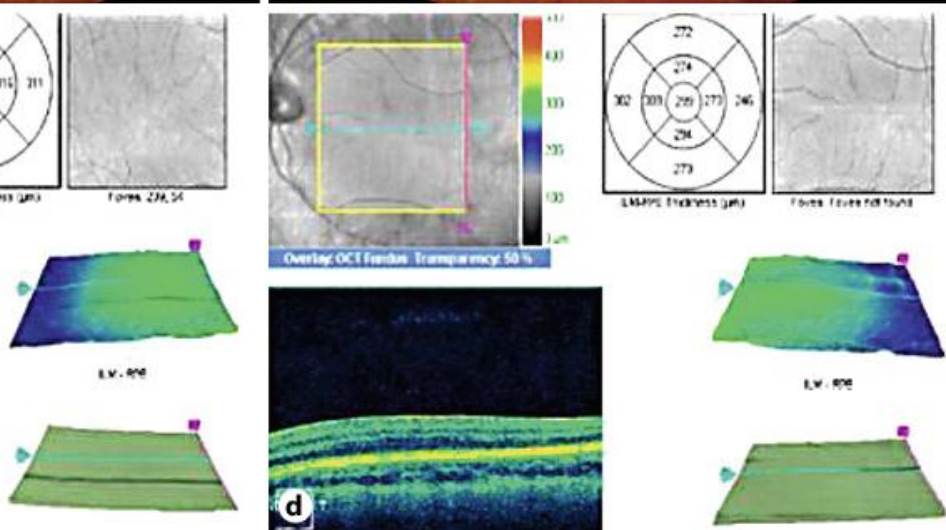

Fig. 1. a, b Fundus photographs of both eyes showing a slightly diffuse depigmentation in the midperiphery and a pinkish optic disk with clear margins and a mild macular dystrophy at the posterior pole. $\mathbf{c}, \mathbf{d}$ OCT images of both eyes showing the absence of normal macular depression and high reflectivity across the fovea. 

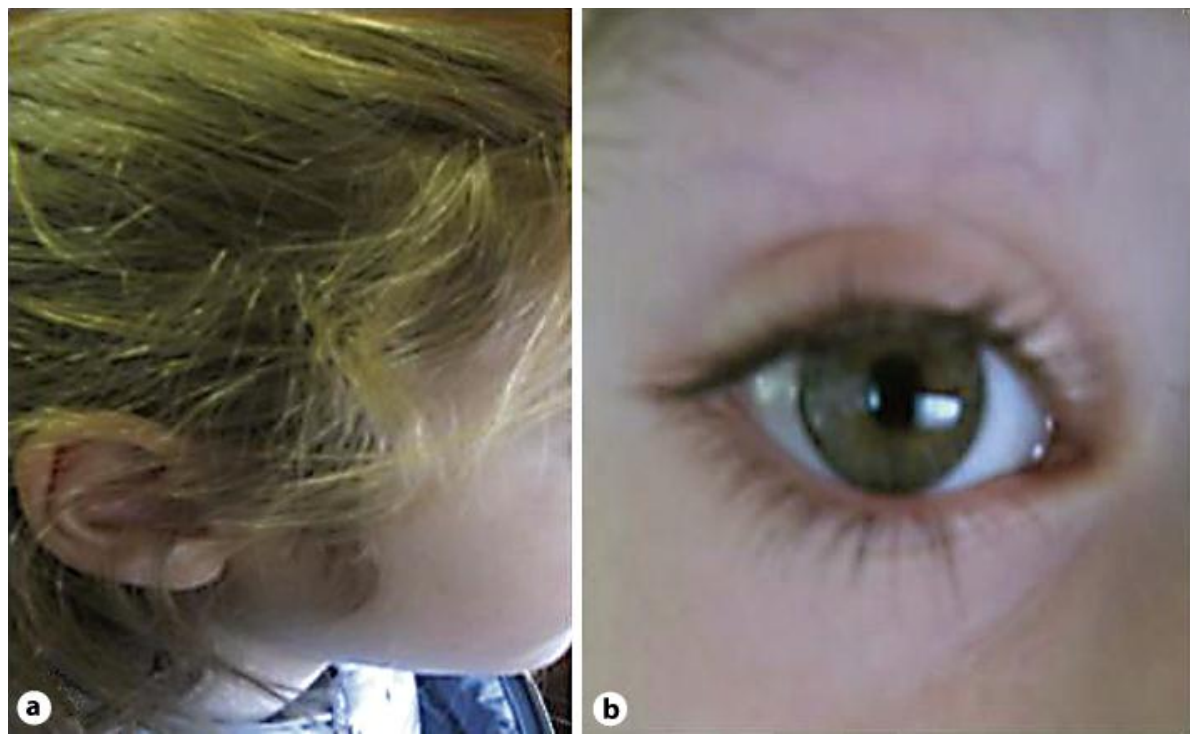

Fig. 2. Pictures showing normal pigmentation of the skin, hair and iris.

\section{References}

1 Grønskov K, Ek J, Brondum-Nielsen K: Oculocutaneus albinism. Orphanet J Rare Dis 2007;2:2-43.

-2 Ergun E, Hermann B, Wirtitsch M, Unterhuber A, Ko TH, Sattmann H, Scholda C, Fujimoto JG, Stur M, Drexler W: Assessment of central visual function in Stargardt's disease/fundus flavimaculatus with ultrahigh-resolution optical coherence tomography. Invest Ophthalmol Vis Sci 2005;46:310-316.

-3 Lenassi E, Jarc-Vidmar M, Glavac D, Hawlina M: Pattern electroretinography of larger stimulus field size and spectral-domain optical coherence tomography in patients with Stargardt disease. Br J Ophthalmol 2009;93:1600-1605.

4 Chong GT, Farsiu S, Freedman SF, Sarin N, Koreishi AF, Izatt JA, Toth CA: Abnormal foveal morphology in ocular albinism imaged with spectral-domain optical coherence tomography. Arch Ophthalmol 2009;127:37-44.

-5 Meyer CH, Lapolice DJ, Freedman SF: Foveal hypoplasia in oculocutaneous albinism demonstrated by optical coherence tomography. Am J Ophthalmol 2002;133:409-410.

6 Coker JG, Duker JS: Macular disease and optical coherence tomography. Curr Opin Ophthalmol 1996;7:33-38.

7 Giovannini A, Amato GP, Mariotti C, Scassellati-Sforzolini B: OCT imaging of choroidal neovascularization and its role in the determination of patients' eligibility for surgery. Br J Ophthalmol 1999;83:438-442.

-8 Hee MR, Puliafito CA, Duker JS, Reichel E, Coker JG, Wilkins JR, Schuman JS, Swanson EA, Fujimoto JG: Topography of diabetic macular edema with optical coherence tomography. Ophthalmology 1998; 105:360-370

-9 Hirakawa H, Iijima H, Gohdo T, Tsukahara S: Optical coherence tomography of cystoid macular edema associated with retinitis pigmentosa. Am J Ophthalmol 1999;128:185-191.

10 Izquierdo NJ, Emanuelli A, Izquierdo JC, García M, Cadilla C, Berrocal MH: Foveal thickness and macular volume in patients with oculocutaneous albinism. Retina 2007;27:1227-1230.

11 Craft JL, Albert DM, Fulton AB: Human albinism, light and electron microscopy studies. Arch Ophthalmol 1978;96:305-310.

Drs. S. Rossi and F. Testa contributed equally to this article. 